\title{
EL FENÓMENO CLÍNICO GUT FEELINGS, ¿CÓMO EXPRESARLO EN ESPAÑOL MEXICANO? UN ESTUDIO MULTIMÉTODO
}

Ismael Ramírez-Villaseñor ${ }^{*}$, Alberto Javier Gutiérrez-Castillo2 , Demetrio SalvadorVázquez-Vázquez³ Perla Xochipilli Arce-Jiménez ${ }^{4}$ y Guadalupe Palmira Ramírez-Gutiérrez ${ }^{5}$

'Profesor de Medicina Familiar y Farmacología Aplicada, Escuela de Medicina y Salud, Tecnológico de Monterrey, Campus Guadalajara; 2Profesor de asignatura, Escuela de Ciencias de la Salud, Universidad del Valle de México, Campus Zapopan. Zapopan, Jal.; ${ }^{3}$ Unidad de Medicina Familiar con Hospital 19 (UMFH 19), IMSS, Delegación Jalisco; ${ }^{4}$ Unidad de Medicina Familiar 97, IMSS, Magdalena, Jal.; 5 Médico familiar, Unidad de Medicina Familiar N.o 92, IMSS, Delegación Jalisco. México

RESUMEN: Introducción: Los sentidos de alarma y seguridad conocidos en la literatura médica como gut feelings (GF) han sido validados por médicos familiares (MF) de diversos países. Material y métodos: El presente trabajo difundió el concepto GF en tres ciudades mexicanas buscando conocer los términos preferidos por los MF para describirlo. Se hicieron encuestas en dos fases; en la segunda fase la encuesta se modificó a partir de los datos de la primera. En el proceso entre las fases y el análisis se recurrió a procesos hermenéuticos dentro del equipo investigador. Resultados: El 96\% de los participantes reconoció haber percibido el sentido de alarma y el $88 \%$ el sentido de seguridad. Los términos preferidos para GF fueron presentimiento clínico e intuición somática pronóstica. El primero fue preferido por los médicos de mayor edad. Discusión: La traducción literal de gut feelings («sentimientos intestinales») se descartó en la primera fase. La segunda fase mostró clara preferencia por presentimiento clínico. Conclusiones: GF en español mexicano podría describirse como presentimiento clínico. El proceso del estudio y la discusión minuciosa de los resultados hizo recordar a los investigadores casos personales en los que el presentimiento clínico jugó un papel esencial, lo que al parecer cambió el significado de la experiencia recordada. Enseñar el concepto de presentimiento clínico podría tener efectos semejantes en la comunidad de medicina familiar.

Palabras clave: Educación médica. Emociones. Competencia clínica.
THE CLINICAL PHENOMENON, "GUT FEELINGS". HOW DO WE EXPRESS THIS IN MEXICAN SPANISH? A MULTIMETHOD STUDY

ABSTRACT: Introduction: The feelings of alarm and safety known in the medical literature as "Gut feelings" (GF) have been validated by family physicians (FP) in diverse countries. Material and methods: This work disseminated the concept of GF in 3 Mexican cities to know the terms preferred by family physicians to describe it. Questionnaires were applied in two phases; in the second phase, the questionnaire was modified using data from the first. During the process between phases and analysis, hermeneutic processes within the research team were used. Results: Of the participants, $96 \%$ recognized having perceived alarm and $88 \%$, a sense of safety. The preferred names for GF were: clinical premonition, and somatic intuition of prognosis. The former was preferred by older physicians. Discussion: The literal translation of gut feelings (intestinal feelings) was discarded in the first phase. In phase 2 , there was a clear preference for "clinical feeling". Conclusions: GF in Mexican Spanish can be described as "clinical feeling". The study and detailed discussion of the results, reminded the researchers of personal cases where clinical judgment played an essential role. This seemed to change the meaning of the remembered experience. Teaching the concept of "clinical feeling" could have similar effects in the family medicine community. (Rev Mex Med Fam. 2018;5:62-7) Corresponding author: Ismael Ramírez-Villaseñor, ismaelravi@gmail.com

Key words: Medical education. Emotions. Clinical competence.

\section{Correspondencia:}

*Ismael Ramírez-Villaseñor

Remanso del Jabalí, 133,

Col. Ciudad Bugambilias

C.P. 45238, Zapopan, Jal., México

E-mail: ismaelravi@gmail.com 


\section{INTRODUCCIÓN}

Stolper, et al. han demostrado que los médicos familiares (MF) experimentados desarrollan un «sentido de alarma» y un «sentido de seguridad» ${ }^{1}$ que funciona como una especie de brújula que permite atender decenas de pacientes en un tiempo reducido, pero con altos niveles de seguridad. El «sentido de alarma» se manifiesta en sensaciones corporales desagradables o pensamientos fugaces de que «algo no está bien con este paciente», aun sin tener claridad del diagnóstico biomédico o las causas de tal sensación ${ }^{2,3}$. Este «aviso» de orden tácito llega a la conciencia del médico haciéndolo ir más lento en el proceso de atención de casos específicos. El «sentido de seguridad», por el contrario, es una sensación de que "todo encaja en el caso del paciente». Ambas sensaciones constituyen el concepto de gut feelings (GF) ${ }^{3}$, el cual ha sido validado a través de un instrumento disponible en inglés, francés, alemán y polaco, además del original en holandés ${ }^{4}$. Este fenómeno ha sido incorporado al sistema legal holandés como parte de las competencias clínicas de los MF. Los GF podrían ser esenciales para diagnosticar oportunamente enfermedades graves y de escasa incidencia, como el tromboembolismo pulmonar ${ }^{5,6}$. A partir de estos hechos, se decidió emprender la difusión del concepto GF entre los MF mexicanos. Al hacerlo, se comprendió que era necesario expresar en español mexicano las ideas de «sentido de seguridad»y «sentido de alarma». El proyecto se empezó a explorar en noviembre de 2016.

\section{LAS EMOCIONES, EL CONOCIMIENTO TÁCITO Y LA TOMA DE DECISIONES}

«... Los médicos no basan sus decisiones solamente en argumentos puramente racionales... y heurísticos... ambos están bajo la influencia de emociones» ${ }^{7}$. Para Damasio, las emociones son sensaciones somáticas agradables o desagradables, y los sentimientos constituyen una interpretación más elaborada de las primeras, que pueden ser expresadas más elaboradamente. Las emociones y los sentimientos están presentes todo el tiempo, y serían el recuerdo de razonamientos heredados por la evolución filogenética y el desarrollo ontogénico de la persona. Se nace con un equipamiento básico de emociones y se sigue guardando aquéllas y los sentimientos que han sido decisivos en la historia personal ${ }^{8}$. Es decir, se guarda una memoria de los contextos que han sido importantes en nuestra vida. Los GF se explicarían como recuerdos de nuestra experiencia clínica. Polanyi demostró la existencia de una forma de conocimiento que no puede ser expresado en palabras. Usó el ejemplo del reconocimiento facial. «... Ordinariamente no podemos decir cómo es que reconocemos una cara que conocemos. No lo podemos poner en palabras. Pero con ayuda de la técnica policíaca, escogemos partes de un rostro, nariz, pelo, boca, y armamos una imagen más o menos cercana a la que deseamos comunicar. Pero no podemos decir cómo lo hicimos. De igual forma, reconocemos los estados de ánimo del rostro humano, sin poder decir exactamente por qué signos lo sabemos...»". El conocimiento tácito conecta dos términos, y cuando comprendemos la relación entre ambos, su unidad integral nos permite comprender la entidad completa cuando nuestra conciencia pone atención en el significado del conjunto 9 . El conocimiento tácito opera sobre una acción interna que somos incapaces de controlar o incluso de sentir. Nuestro cuerpo es el instrumento último de todo nuestro conocimiento externo, sea este intelectual o práctico ${ }^{9}$. El fenómeno GF parece ser una expresión de esta forma de conocimiento derivado de la experiencia clínica. Y no habría solución a los problemas de la realidad compleja sin la participación del conocimiento tácito. 


\section{OBJETIVO}

Obtener una término o frase que describa en español mexicano el concepto clínico GF.

\section{MATERIAL Y MÉTODOS}

Durante las Quintas Jornadas Médicas de la Asociación de Médicos Familiares de Baja California Sur, el 13 de noviembre de 2017, se presentó la conferencia «Los sentimientos intestinales y el pronóstico del paciente», y los médicos presentes completaron -de forma voluntaria y anónima- una encuesta semiestructurada que preguntaba si habían sentido «el sentido de alarma y el de seguridad» y qué nombre preferían para expresarlo en español mexicano. Se dieron cuatro opciones: a) sentimientos intestinales; b) sentido de alarma y de seguridad; c) intuición somática, y d) olfato clínico, más una opción abierta. Se obtuvieron 25 encuestas contestadas (100\%). Se hizo un primer análisis, y posteriormente, en febrero de 2017, se realizó el mismo procedimiento en un evento académico del Colegio Jalisciense de Medicina Familiar, Capítulo Sur, en Ciudad Guzmán, y se obtuvieron 14 encuestas completadas (100\%). Esto comprendió la primera fase del estudio. El análisis de las 39 encuestas de la primera fase motivó hacer cambios en la encuesta de la segunda fase, que se produjo en Guadalajara, Jalisco, el 30 de septiembre de 2017. Las opciones fueron: a) intuición somática pronóstica; b) presentimiento clínico, y c) opción abierta.

Se obtuvieron 19 encuestas completadas, de 20 posibles. Se volvió a hacer un análisis estadístico con el programa EPINFO, versión EE.UU. 7.2.0.1 y XLSTAT 2017.5. El equipo investigador se reunió para llegar a un consenso mediante discusión libre y pormenorizada de todo el proceso. Se incluyó un análisis sobre definiciones en inglés y español del concepto GF. De especial relevancia fue la traducción del polaco al inglés y de éste al español de la palabra przeczucie,
Tabla 1. Porcentaje de preferencias para denominar GF en la primera fase del estudio

\begin{tabular}{|l|c|c|}
\hline & $\mathbf{f x}$ & $\%$ \\
\hline Intuición somática & 11 & 28 \\
\hline Olfato clínico & 9 & 24 \\
\hline $\begin{array}{l}\text { Sentido de alarma y } \\
\text { sentido de seguridad }\end{array}$ & 8 & 20 \\
\hline Corazonada & 8 & 20 \\
\hline $\begin{array}{l}\text { Instinto médico, intuición clínica, } \\
\text { sentimientos intestinales }\end{array}$ & 3 & 8 \\
\hline Total & 39 & 100 \\
\hline
\end{tabular}

$\mathrm{fx}$ : frecuencias.

Tabla 2. Porcentaje de preferencias para denominar GF en español mexicano en la segunda fase

\begin{tabular}{|l|c|c|}
\hline & $\mathbf{f x}$ & $\%$ \\
\hline Presentimiento clínico & 12 & 61 \\
\hline Intuición somática pronóstica & 7 & 39 \\
\hline Total & 19 & 100 \\
\hline
\end{tabular}

fx: frecuencias.

ocurrida en la validación al polaco del Gut Feelings Questionnaire (GFQ) ${ }^{4}$. De este modo en el presente trabajo se incluyeron elementos hermenéuticos, en integración con datos cuantitativos por lo que el presente trabajo constituye una investigación mixta o multimétodo ${ }^{10-12}$.

\section{RESULTADOS}

La tasa de respuesta de las encuestas fue del 97.5\%. El 96.4\% de los participantes afirmaron haber experimentado «el sentido de alarma», mientras que el $88 \%$ percibieron el «sentido de seguridad». La participación de mujeres fue del $69 \%$. Los términos preferidos para denominar el fenómeno GF en la primera fase fueron: intuición somática, olfato clínico, sentido de alarma y sentido de seguridad, corazonada, instinto médico, intuición clínica y sentimientos intestinales (Tabla 1). En la segunda fase, con la encuesta modificada, el orden final de frecuencias fue: presentimiento clínico (61\%) e intuición somática pronóstica (33\%) (Tabla 2). La preferencia por un término u otro 
Tabla 3. Edad del médico y preferencia para denominar GF

\begin{tabular}{|l|c|c|c|c|}
\hline \multirow{2}{*}{} & \multicolumn{2}{|c|}{$\leq \mathbf{3 6}$ años } & \multicolumn{2}{c|}{$\geq \mathbf{3 7}$ años } \\
\cline { 2 - 5 } & $\mathbf{f x}$ & $\%$ & $\mathbf{f x}$ & $\%$ \\
\hline $\begin{array}{l}\text { Intuición somática } \\
\text { pronóstica }\end{array}$ & 8 & 39 & 0 & 0 \\
\hline Presentimiento clínico & 5 & 28 & 6 & 33 \\
\hline Total & 13 & 67 & 6 & 33 \\
\hline
\end{tabular}

$f x$ : frecuencias. $p$ de Fisher $=0.03 \cdot n=19$.

Tabla 4. Años de experiencia profesional y preferencia para denominar GF

\begin{tabular}{|l|c|c|c|c|c|c|}
\hline & \multicolumn{3}{|c|}{$\leq \mathbf{9}$ años } & \multicolumn{2}{|c|}{$\geq \mathbf{1 0}$ años } & \multicolumn{2}{|c|}{ Total } \\
\hline & $\mathbf{f x}$ & $\mathbf{\%}$ & $\mathbf{f x}$ & $\%$ & $\mathbf{f x}$ & $\%$ \\
\hline $\begin{array}{l}\text { Intuición somática } \\
\text { pronóstica }\end{array}$ & 7 & 33 & 1 & 6 & 8 & 39 \\
\hline $\begin{array}{l}\text { Presentimiento } \\
\text { clínico }\end{array}$ & 5 & 28 & 6 & 33 & 11 & 61 \\
\hline Total & 12 & 61 & 7 & 39 & 19 & 100 \\
\hline
\end{tabular}

fx: frecuencias. p de Fisher $=0.05$.

no tuvo relación estadística con el sexo del médico, pero sí con la edad, pues a mayor edad hubo mayor preferencia por presentimiento clínico (chi cuadrada con $\mathrm{p}=0.01$ y prueba de Fisher de $\mathrm{p}=0.03$ ) (Tabla 3 ). Aunque los años de experiencia, agrupados con punto de corte de 10 años, no lograron significación estadística, la prueba de Fisher obtuvo una $\mathrm{p}=0.05$ y Chi cuadrada de $\mathrm{p}=0.08$ (Tabla 4$)$.

\section{DISCUSIÓN}

La primera fase mostró que los MF de dos regiones mexicanas reconocían el fenómeno $\mathrm{GF}^{1,2}$, y que la traducción literal «sentimientos intestinales» podía ser descartada como opción. El equipo investigador reconoció que corazonada, intuición clínica e instinto médico podían fusionarse con el inciso D, olfato clínico, dado el significado de presentir: intuir, tener la impresión de que algo va a suceder. Es adivinar algo antes de que suceda por indicios o señales ${ }^{13}$. Surgió el consenso de incluir en la encuesta de la segunda fase presentimiento clínico y la opción más votada en la primera fase, intuición somática, con el modificador pronóstica para darle connotación clínica. En la decisión influyó que médicos polacos prefirieron presentimiento en lugar de intuición, como ya fue descrito ${ }^{4}$.

Se consideró que, si la hipótesis era correcta, una opción abierta no produciría nuevas opciones relevantes en la siguiente fase. También se consensó que GF en la cultura anglófona es usado para referirse a un sentimiento o reacción basado en una respuesta emocional instintiva, no meditada. Incluye intuición, corazonada, sentimiento, a veces sentido corporalmente, como gut instinct ${ }^{14}$. Stolper, et al. descubrieron que «el sentido de alarma» era expresado por médicos generales de 18 países como una sensación desagradable, algunas veces referida somáticamente, por lo que empezaron a usar la frase inglesa gut feelings en sus comunicaciones ${ }^{15}$.

\section{LIMITACIONES}

Los participantes del presente trabajo representan al subgrupo de MF afiliados a organismos académicos. No se puede afirmar que sea extensivo a toda la comunidad de MF mexicanos. Se usa el término español mexicano, y no castellano, porque para los lingüistas mexicanos es más apropiado el primero ${ }^{16}$. Se consideró que otras lenguas del mundo denominan a la lengua predominante en México como espagnole, spagnuola, Spanish, Spanisch ${ }^{17}$. Este trabajo no se propuso dar luz sobre factores que disparan y modulan los GF, como han hecho otros investigadores ${ }^{18}$.

\section{ASPECTO CUALITATIVO}

En las sesiones de trabajo del equipo investigador surgió la narración de diversas experiencias clínicas personales que parecieron adquirir nueva relevancia a la luz de lo investigado en este proceso:

- Una paciente embarazada acudió a las 04:30 de la madrugada por mareos y 
cefalea moderada al servicio de urgencias de una unidad de medicina familiar (UMF). El médico tuvo una sensación desagradable y, debido a ella y a pesar de la ausencia de signos físicos de gravedad, envió a la paciente al hospital. La paciente desarrolló eclampsia, pero tanto ella como su bebé superaron el episodio con buena salud.

- Otro investigador narró dos casos de consultas a domicilio de niños con dolor abdominal. El primero ya había sido visto en un hospital y no quería volver a él, y el segundo era una niña que había sido vista dos veces en urgencias de la UMF. El médico supo por la abuela de la niña que la madre de ésta le regañaba por quejarse de dolor. En ambos casos el médico sintió que algo no estaba bien y los envió de nuevo al hospital. Ambos niños casi mueren por complicaciones de apendicitis.

- Una MF vio de reojo a una paciente que llegó a su consulta mientras estaba atendiendo a otra paciente, pues sintió un impulso de atender de inmediato a la recién llegada. Así lo hizo y la envió al hospital, donde la paciente murió de hemorragia cerebral. Posteriormente, la MF reflexionó que percibió a una mujer de alrededor de 50 años que mostraba una de sus mamas en la sala de espera, "como si hubiera perdido el pudor; algo muy malo le debería estar pasando».

- Un hombre de 56 años es visto por tos y edema del brazo izquierdo de dos semanas de evolución. Un residente de MF (RMF) sintió que algo andaba muy mal, pero no tenía datos objetivos. Lo envió al internista y éste al angiólogo. El paciente retornó al RMF con diagnóstico de trombosis de la vena subclavia. EL RMF siguió sintiendo que algo no encajaba, le encontró un ganglio supraclavicular y propuso biopsia. El paciente prefirió el plan del angiólogo y murió 10 meses después por complicaciones de cáncer pulmonar.

Los casos descritos describen la presencia de presentimientos clínicos que dejaron un aprendizaje significativo en los médicos que los siguieron de principio a fin, pero que ahora tenían un significado más trascendente para sus médicos. Esta implicación de la experiencia clínica ha sido descrita como: «conforme el médico atiende a más personas y resuelve sus casos, va integrando una base de conocimiento que tendrá disponible para casos futuros» ${ }^{19}$.

\section{CONCLUSIONES}

Los MF encuestados en su mayoría reconocieron haber experimentado el sentido de alarma y seguridad, y algunos expresaron espontáneamente la relevancia que les había significado: «... muchas veces me he dejado llevar por mi instinto con muy buenos resultados... Nunca me imaginé que muchos colegas también lo hubieran experimentado». El equipo de investigadores llegó al consenso de proponer a la comunidad de medicina familiar mexicana el término presentimiento clínico para designar el fenómeno GF. El proceso de investigación hizo recordar a los investigadores casos en los que su presentimiento clínico jugó un papel esencial en el resultado clínico, pero ahora el recuerdo adquirió mayor legitimidad. Enseñar el concepto de presentimiento clínico podría tener efectos semejantes entre los MF.

\section{RECOMENDACIONES}

Sería bienvenido un estudio amplio sobre los factores que impulsan y desarrollan el presentimiento clínico, así como la validación en español mexicano del $G F Q^{20}$. Es necesario investigar la precisión del presentimiento clínico en el pronóstico de problemas agudos y si tiene algún papel en el pronóstico a mediano y largo plazo.

\section{CONFLICTOS DE INTERÉS}

Los autores declaran que no existen conflictos de intereses que influyan en el presente trabajo. 


\section{FUENTE DE FINANCIACIÓN}

Recursos propios de los autores.

\section{PRESENTACIÓN PREVIA}

\section{Ninguna.}

\section{BIBLIOGRAFÍA}

1. Stolper $E$, van Bokhoven $M$, Houben $P$, van Royen $P$, van de Wiel $M$, van der Weijden $T$, et al. The diagnostic role of gut feelings in general practice. A focus group study of the concept and its determinants. BMC Fam Pract. 2009;10:17.

2. Stolper $E$, van Royen $P$, van de Wiel M, van Bokhoven $M$, Houben $P$, van der Weijden $T$, et al. Consensus on gut feelings in general practice. BMC Fam Pract. 2009;10:66.

3. Stolper $E$, van de Wiel $M$, van Royen $P$, van Bokhoven $M$, van der Weijden T, Dinant GJ. Gut feelings as a third track in general practitioners' diagnostic reasoning. J Gen Intern Med. 2011;26(2):197-203.

4. Barais, M, Hauswaldt, J, Hausmann, D, Czachowski, S. The linguistic validation of the gut feelings questionnaire in three European languages. BMC Family Practice. 2017;18(1):54.

5. Stolper CF, Legemaate J, Dinant GJ. How do disciplinary tribunals judge the 'gut feelings' of doctors? An analysis of Dutch tribunal decisions 2000-2008. J of Law and Med. 2010;18(1):68-75

6. Barais M, Morio N, Cuzon Breton A, Barraine P, Calvez A. I Can't Find Anything Wrong: It Must Be a Pulmonary Embolism: Diagnosing Suspected Pulmonary Embolism in Primary Care, a Qualitative Study. PLoS One. 2014;9(5):e98112.
7. Reach G. Simplistic and complex thought in medicine: the ratio nale for a person-centered care model as a medical revolution. Patient Prefer Adherence. 2016;10:449-57.

8. Damasio AR. Emotions and Feelings. En: Descartes' Error Emotion, Reason, and the Human Brain. New York: Avon Books 1994. p. 127-64.

9. Polanyi M. The tacit dimension, with a new foreword by Amartya K

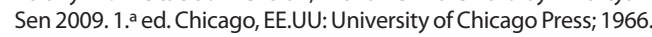

10. Creswell JW, Fetters MD, Ivankova NV. Designing a mixed method study in primary care. Ann Fam Med. 2004:2(1):7-12.

11. Borkan JM. Mixed methods studies: A foundation for primary care research. Ann Fam Med. 2004;2(1):4-6.

12. Stange KC. Multimethod research. Ann Fam Med. 2004;2(1):2-3

13. Dleraees. [Online]. Disponible en: http://dle.rae.es/?id=U6BiEWD. [Acceso el 30 de octubre de 2017].

14. Lingueees. [Online]. Disponible en: ttps://www.linguee.es/ingles-espanol/traduccion/gut feeling.html. [Acceso el 31 de octubre de 2017].

15. Stolper E. The 'Sense of Alarm' ('Gut Feeling') in clinical practice A survey among European general practitioners on recognition and expression. Eur J Gen Pract. 2010;16(2):72-4.

16. Procesocommx. Proceso. [Online]. Disponible en: http://www. proceso.com.mx/383868/la-lengua-oficial-en-mexico-no-es-elespanol. [Acceso el 30 de octubre de 2017].

17. Ilceedumx. [Online]. Disponible en: http://bibliotecadigital.ilce. edu.mx/sites/fondo2000/vol1/algunas-minucias/html/17.html. [Acceso el 30 de octubre de 2017].

18. Oliva B, March S, Gadea C, Stolper E, Esteva M. Gut feelings in the diagnostic process of Spanish GPs: a focus group study. BMJ Open. 2016;6(E012847).

19. McWhinney IR. 'An acquaintance with particulars...' Fam Med. 1989;21(4):296-8. Citado por Malterud K. The art and science of clinical knowledge: evidence beyond measures and numbers. Lancet. 2001:358:397-9.

20. Stolper CF, van de Wiel MW, de Vet $H C$, Rutten $A L$, van Royen $P$, van bokhoven MA, et al. Family physicians' diagnostic gut feelings gut feelings are measurable: Construct validation of a questionnaire. BMC Fam Pract. 2013;14(1). 